\title{
Efeito da escala MELD na mortalidade após transplante de fígado*
}

\author{
The effect of MELD on the post liver \\ transplantation mortality rate*
}

\author{
Jorge Henrique Fernandes*, Lincoln Saito Millan*, \\ Paulo Celso Bosco Massarollo**, Sérgio Mies ${ }^{\star \star \star}$
}

Fernandes JH, Millan LS, Massarollo PCB, Mies S. Efeito da escala MELD na mortalidade após transplante de fígado. Rev Med (São Paulo) 2002 jan./dez.;81(1/4):15-21.

RESUMO: A alta mortalidade na lista de espera por transplante de fígado (Tx) tem estimulado a adoção de critérios de gravidade para alocação de órgãos. O objetivo desse trabalho é investigar qual o efeito da classificação MELD (Model for End-Stage Liver Disease) na mortalidade após o Tx. Foram revisados 237 casos de Tx eletivo com doador cadáver realizados entre 1\%/11/1995 e 30/7/2001. Na análise de regressão logística, foi encontrada uma interação significante entre a pontuação MELD e a mortalidade nos primeiros 6 meses após o transplante (odds ratio $=1,052 ;$ IC $95 \%=1,015$ a 1,090; $p=0,02$ ). Para estudar o efeito da escala MELD na sobrevida atuarial, os pacientes foram divididos em dois grupos: Grupo A ( $n=126)$ com valores de MELD abaixo da média da casuística global $(14,4)$ e Grupo $B(n=111)$ com pontuação MELD acima desse valor. Existe uma diferença significante entre a sobrevida ao longo do tempo nos dois grupos $(p=0,0374)$. A análise de regressão proporcional de Cox indicou risco 1,65 vezes maior no grupo $B$ (risco relativo $=1,6523 ;$ IC95\% $=1,0240$ a 2,6662).

DESCRITORES: Transplante de fígado/mortalidade; Escalas; Obtenção de órgãos/normas.

\section{INTRODUÇÃO}

O transplante de fígado ( $T x$ ) é hoje preconizado como conduta de eleição em doenças de fígado terminais, sejam elas crônicas ou agudas. Esta opção baseia-se nos bons resultados atuais do procedimento, cuja taxa de sobrevida após um ano subiu de aproximadamente $70 \%$ no começo da década de 1980 para 80 a $90 \%$ no final da década de $1990^{7}$. Atualmente, a taxa de sobrevida de cinco anos ultrapassa $60 \%{ }^{7}$.

Habitualmente, a evolução dos pacientes submetidos a $T x$ refere-se à probabilidade de sobrevida em função do tempo decorrido após o procedimento. Porém, considerando-se que a demanda por órgãos excede a oferta, a probabilidade de sobrevida dos candidatos a Tx não depende apenas de como eles respondem à intervenção cirúrgica, mas também sofre influência de como eles evoluem durante o período em que aguardam por um enxerto a partir da indicação. O Estado de São Paulo registrou, dentre os pacientes transplantados no ano de 2001, um tempo médio de espera de 19,5 meses ${ }^{18}$. Durante este período, existe uma taxa de mortalidade que é diretamente proporcional ao tempo de espera.

A mortalidade na lista de espera depende não apenas do tempo decorrido desde a inclusão, mas

* Prêmio Oswaldo Cruz 2002 (Área Cirúrgica). XXI COMU Congresso Médico Universitário da FMUSP.

** Acadêmicos do 3o ano do Curso de Graduação da FMUSP.

${ }^{* * *}$ Professor Doutor da Disciplina de Cirurgia Geral e Topografia Estrutural Humana - (Orientador).

**** Professor Associado da Disciplina de Cirurgia Geral e Topografia Estrutural Humana (Orientador).

Endereço para correspondência: Lincoln Saito Millan, R: Te. Ubirajara Monory, 197 - Pq Jabaquara, CEP: 04345-020

São Paulo, SP.e-mail: Ismillan@yahoo.com 
também da condição clínica do paciente nesse momento. Esse fato decorre da heterogeneidade dos candidatos, que podem ser divididos em grupos distintos, conforme a gravidade da doença. Com o objetivo de classificar os pacientes segundo o risco de mortalidade em lista, foram propostos alguns modelos que pretendem avaliar a gravidade e o prognóstico da doença ${ }^{11}$.

Existem hoje dois modelos mais citados para avaliação de gravidade para doenças hepáticas: a classificação de Child-Turcotte-Pugh (CTP) e, mais recentemente, a escala MELD (Model for End-Stage Liver Disease) ${ }^{11}$. A primeira, criada inicialmente para se avaliar risco cirúrgico de pacientes com cirrose, tem como variáveis as concentrações séricas de albumina e de bilirrubina, o tempo de protrombina e as condições clínicas de ascite e encefalopatia ${ }^{7,11}$. A escala MELD, um modelo criado inicialmente para estimar a sobrevida de pacientes submetidos a desvio venoso (shunt) portossistêmico intra-hepático transjugular (TIPS), tem como variáveis os níveis séricos de creatinina $(\mathrm{Cr})$ e bilirrubina total $(\mathrm{BT})$ e a razão internacional normalizada para o tempo de protrombina (INR), podendo-se utilizar também a etiologia da doença ${ }^{11}$. Já se demonstrou que a escala MELD estima melhor a gravidade da doença hepática em candidados a Tx do que a CTP ${ }^{3}$.

A disponibilidade desses modelos estimula a adoção de critérios de gravidade para alocação de enxertos, priorizando os pacientes com menor expectativa de sobrevida. Dessa forma, pretende-se diminuir o número de mortes na lista de espera ${ }^{9}$. Com esse objetivo, a UNOS (United Network for Organ Sharing) adota, desde to de fevereiro de 2002, a escala MELD para priorização na lista de Tx dos EUA $^{19}$.

Um aspecto pouco estudado refere-se às potenciais conseqüências adversas da adoção desse critério de alocação de enxertos sobre os resultados do tratamento. De fato, a maior mortalidade dos pacientes mais graves pode ser esperada não somente antes mas também depois do Tx. Esse efeito foi observado, por exemplo, em casos com pior função renal ${ }^{13}$. Foi demonstrado que pacientes com depuração de $\mathrm{Cr}<40 \mathrm{~mL} / \mathrm{min}$ apresentam maior mortalidade 30 dias e dois anos após o Tx ${ }^{13}$. Assim, é possível que a utilização de critérios de gravidade não reduza a mortalidade mas apenas a transfira para depois do tratamento. Entretanto, ainda existem poucas investigações sobre a capacidade da escala MELD de prever a sobrevida pós-transplante ${ }^{3}$.

O objetivo do presente estudo é analisar o efeito da escala MELD na mortalidade após o Tx.

\section{CASUÍSTICA E MÉTODO}

Foram estudados retrospectivamente todos os
376 Tx realizados pela Unidade de Fígado do Hospital das Clínicas da Faculdade de Medicina da Universidade de São Paulo (HCFMUSP) no período de $1^{\circ}$ de novembro de 1995 a 30 de julho de 2001. Deste grupo, foram excluídos todos os casos nos quais 0 enxerto foi proveniente de doadores vivos. Esses compreendem 17 transplantes intervivos ${ }^{16}$ e 18 transplantes da modalidade repique ${ }^{1,14}$. Também foram excluídos 25 casos de retransplante, 25 casos de insuficiência hepática aguda grave e 54 casos nos quais não foram encontrados resultados laboratoriais necessários para o cálculo da escala MELD. Desta forma, foram incluídos no estudo 237 pacientes submetidos a Tx eletivo com doador cadáver. As etapas de seleção de pacientes para constituição da casuística são mostradas na Figura 1. Os diagnósticos dos pacientes incluídos são mostrados na Tabela 1.

Tabela 1 - Caracterização dos pacientes: etiologia principal da doença hepática.

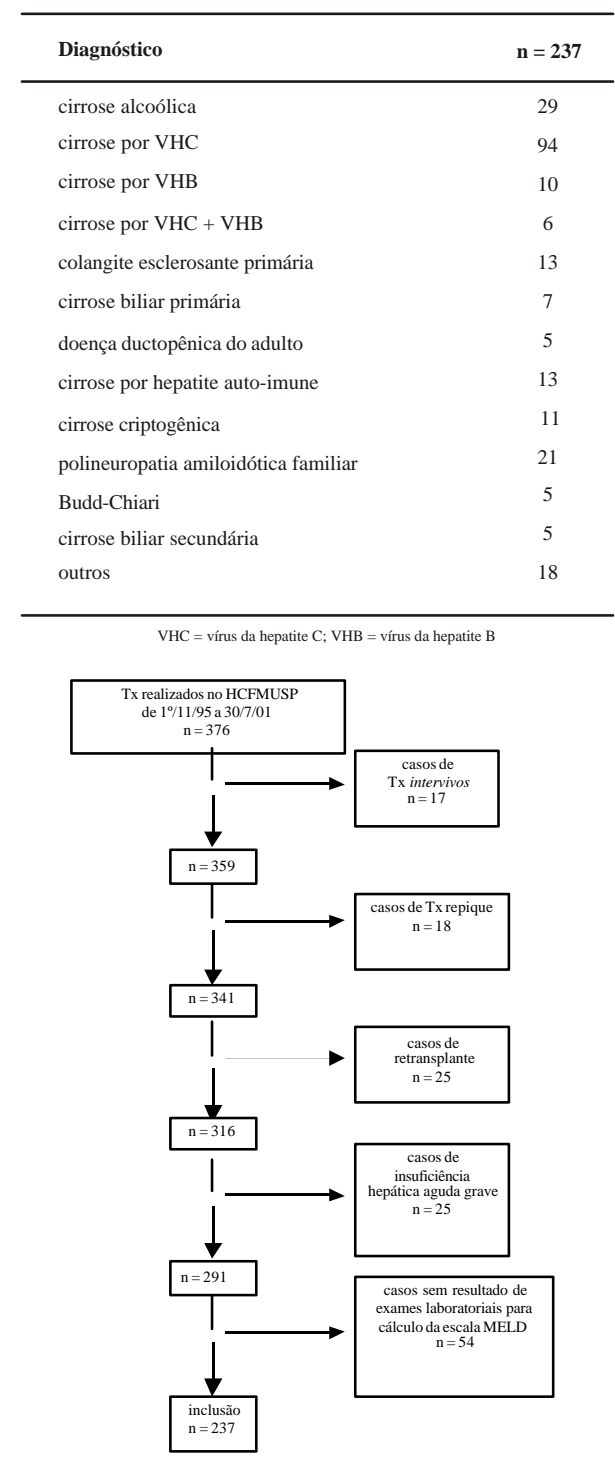

Figura 1 - Diagrama de fluxo mostrando as etapas de seleção de pacientes para constituição da casuística. 
Foram registrados os resultados de INR, $\mathrm{Cr}$ e BT realizados antes do Tx sendo considerados os valores mais próximos da operação. Foram excluídos os pacientes cujo intervalo entre a data dos exames e a data do transplante tenha excedido quatro meses.

A gravidade da doença hepática foi avaliada através da escala MELD, sem se considerar a etiologia da doença ${ }^{11}$. O cálculo foi realizado utilizando a seguinte fórmula:

$$
\operatorname{MELD}=3,8 \ln (B T)+11,2 \ln (\mathrm{INR})+9,6 \ln (\mathrm{Cr})+6,4
$$

A seguir, foi avaliada a situação dos pacientes em 31 de dezembro de 2001. Foram anotadas a data do Tx e, nos que faleceram, a data desse evento.

O efeito dos valores da escala MELD na mortalidade dos primeiros 6 meses após o transplante foi estudado por meio de análise de regressão logística5. A análise do comportamento temporal da sobrevida de pacientes ao longo do período de acompanhamento foi realizada por meio de construção de curvas atuariais de Kaplan-Meier ${ }^{12}$. Para estudo do efeito da escala MELD na sobrevida atuarial, os pacientes foram divididos em dois grupos: Grupo A, que incluiu pacientes com valores de MELD abaixo da média global da casuística; e Grupo $B$, que incluiu pacientes com pontuação MELD acima desse valor. A sobrevida atuarial de pacientes nestes dois grupos foi comparada utilizando-se o teste de Cox-Mantel (logrank $)^{10}$ e o modelo de regressão por tábua de sobrevivência de Cox ${ }^{6}$.

A comparação de variáveis quantitativas entre os grupos A e B foi realizada por meio do teste "t" de Student. A comparação de variáveis qualitativas foi realizada por meio do teste Ç de Pearson com correção de Yates.

Todas as análises estatísticas com exceção da regressão logística foram realizadas utilizando o programa SPSS for Windows versão 9.0 (Statistical Package for the Social Sciences Inc., Chicago, IL, EUA). A análise de regressão logística foi realizada utilizando o programa SigmaStat for Windows versão 2.0 (Jandel Corporation, San Rafael, CA, EUA).

Os resultados são expressos em média \pm desvio padrão e em intervalo de confiança de 95\% (IC95\%).

Foi utilizado nível de significância de $5 \%$ $( \pm=0,05)$.

\section{RESULTADO}

Dos 237 pacientes da casuística global, 144 eram do sexo masculino (60,8\%). A média das idades na data do transplante era 46,2 $\pm 13,2$ anos (limites 10 a 75). Os dados demográficos da casuística total e dos grupos A e B são apresentados na Tabela 2. Os dois grupos são semelhantes em relação ao sexo e à idade.
Tabela 2 - Caracterização dos pacientes: dados demográficos.

\begin{tabular}{ccccc}
\hline & $\begin{array}{c}\text { Global } \\
\mathbf{n = 2 3 7}\end{array}$ & $\begin{array}{c}\text { Grupo A } \\
\mathbf{n = 1 2 6}\end{array}$ & $\begin{array}{c}\text { GrupoB } \\
\mathbf{n = 1 1 1}\end{array}$ & $\mathbf{p}$ \\
\hline $\begin{array}{c}\text { Sexo (masculino / feminino) } \\
\text { Idade (anos) }\end{array}$ & $144 / 93$ & $69 / 57$ & $75 / 36$ & 0,06 \\
média & $46,2 \pm 13,2$ & $45,3 \pm 13,1$ & $47,3 \pm 13,2$ & 0,246 \\
limites & $10-75$ & $14-75$ & $10-66$ & - \\
Pontuação MELD & & & & \\
média & $14,4 \pm 7,3$ & $9,4 \pm 3,9$ & $20,1 \pm 6,0$ & - \\
limites & $-6,1-50,3$ & $-6,1-14,4$ & $14,5-50,3$ & - \\
percentil 25 & 10,0 & 7,6 & 15,9 & - \\
percentil 50 & 13,9 & 10,2 & 17,8 & - \\
percentil 75 & 17,4 & 12,3 & 23,0 & - \\
\hline
\end{tabular}

O histograma dos valores de MELD na casuística global é mostrado junto com a curva normal na Figura 2. O valor médio da pontuação MELD da casuística global foi 14,4 $\pm 7,3$ (limites 6,1 a 50,3). Havia 126 pacientes com MELD inferior a 14,4 (Grupo A), e 111 com MELD acima desse valor (Grupo B). A distribuição da pontuação MELD nos grupos $A$ e $B$ é apresentada por meio de gráficos box-plot na Figura 3.

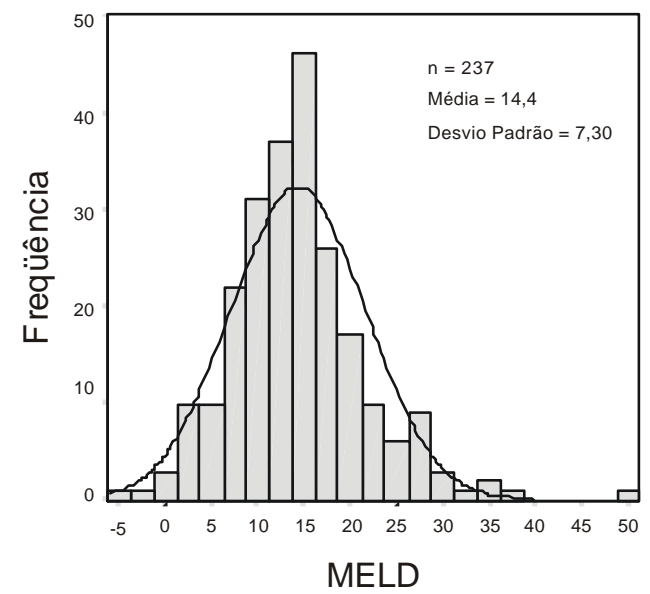

Figura 2 - Histograma mostrando a distribuição dos valores de MELD na casuística global.

$\mathrm{Na}$ análise de regressão logística, foi encontrada uma interação significante entre a pontuação MELD e a mortalidade nos primeiros 6 meses após o transplante (odds ratio = 1,052; IC 95\% $=1,015$ a 1,090). O sumário da análise de regressão logística é apresentado na Tabela 3. A Figura 4 apresenta o gráfico da probabilidade estimada de óbito nos primeiros 6 meses em função da variação da pontuação MELD entre os valores 0 e 50. 


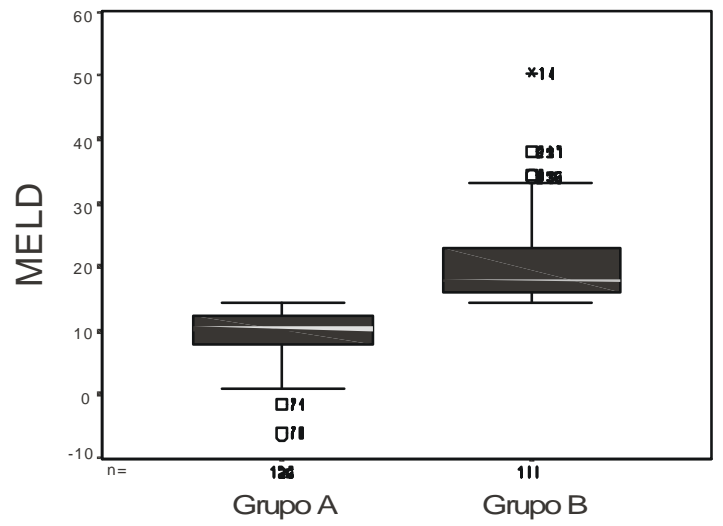

Figura 3 - Gráfico box-plot mostrando a distribuição dos valores de MELD nos pacientes dos Grupos A e B.

Tabela 3 - Sumário da análise de regressão logística entre a pontuação MELD e a mortalidade até 6 meses após o transplante de fígado.

\begin{tabular}{lcccccc}
\hline Variável & $\begin{array}{c}\text { Coeficiente } \\
(\beta)\end{array}$ & $\begin{array}{c}\text { Erro } \\
\text { Padrão }\end{array}$ & $\begin{array}{c}\text { Wald } \\
\left(\chi^{2}\right)\end{array}$ & $\begin{array}{c}\text { Odds } \\
\text { Ratio }\end{array}$ & IC95\% & p \\
\hline constante & $-2,137$ & 0,378 & - & - & - & - \\
MELD & 0,0506 & 0,0216 & 5,521 & 1,052 & 1,015 a 1,090 & 0,020 \\
\hline
\end{tabular}

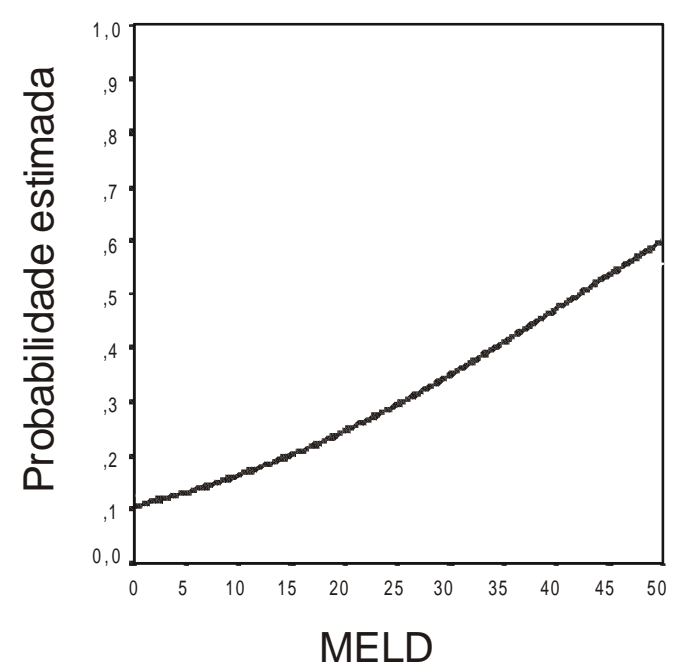

Figura 4 - Gráfico da probabilidade estimada de óbito nos primeiros 6 meses em função da variação da pontuação MELD entre os valores 0 e 50.

As curvas de Kaplan-Meier com a sobrevida atuarial dos pacientes na casuística global e nos grupos A e B são mostradas nas Figuras 5 e 6 . Existe uma diferença estatisticamente significante entre a sobrevida ao longo do tempo nos dois grupos $(p=0,0374)$. O risco cumulativo de óbito em função do tempo nos grupos A e B é apresentado na Figura 7. A análise de regressão proporcional de Cox indicou que os pacientes do grupo $B$ apresentam um risco 1,65 vezes maior de falecer do que os do grupo $A$ (risco relativo $=1,6523 ; \mathrm{IC} 95 \%=1,0240$ a 2,6662).

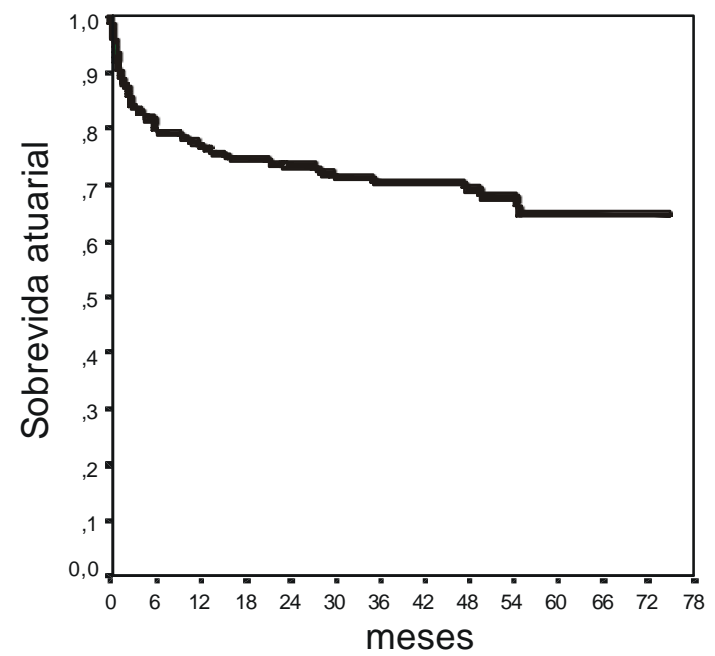

Figura 5 - Curva de Kaplan-Meier mostrando a sobrevida atuarial após o transplante de fígado na casuística global.

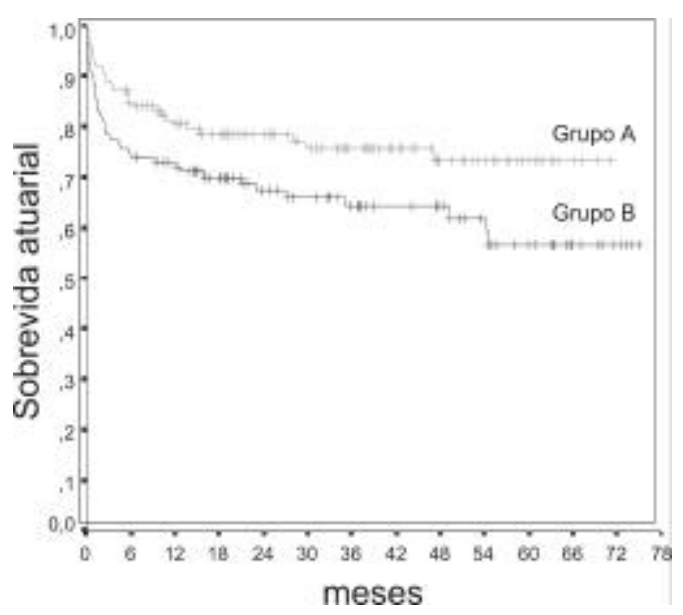

Figura 6 - Curva de Kaplan-Meier mostrando a sobrevida atuarial após o transplante de fígado nos pacientes dos grupos A e B.

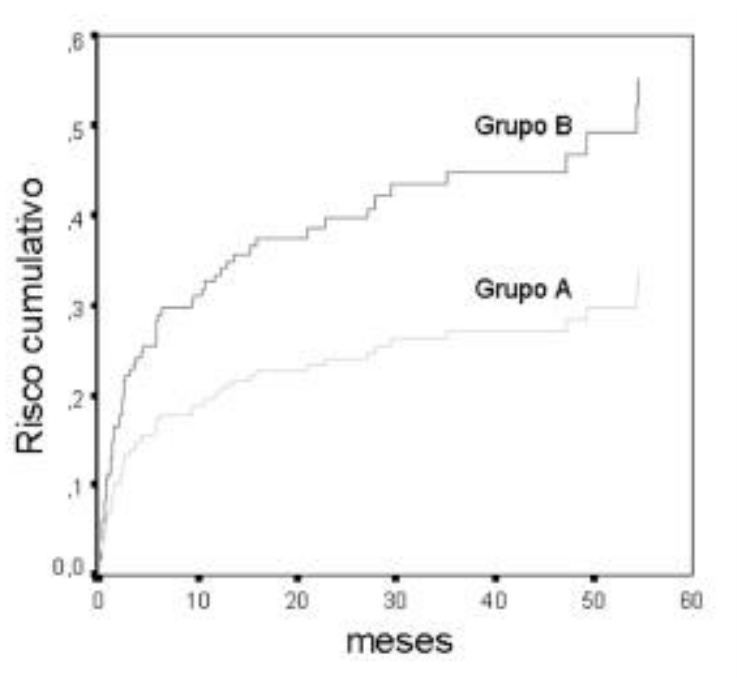

Figura 7 - Curvas de risco cumulativo de mortalidade ao longo do tempo nos pacientes dos grupos A e B. 


\section{DISCUSSÃO}

A alocação dos órgãos obtidos de doadores cadáveres é um dos temas mais polêmicos da área de transplantes. Embora exista consenso sobre a necessidade de oferecer a todos os pacientes igual oportunidade de acesso ao enxerto, há grande controvérsia sobre a melhor maneira de atingir este objetivo $^{8}$. No Brasil, a Lei dos Transplantes, que está em vigor desde de 1997, estabeleceu a distribuição de órgãos segundo a classificação dos candidatos por ordem cronológica de inscrição no cadastro de receptores ${ }^{15}$.

Embora o assunto interesse a todas as modalidades de transplante, a questão assume importância especial no caso do fígado. Diversamente do transplante renal, esta modalidade não dispõe de métodos de suporte vital capazes de prolongar a vida dos pacientes no estágio terminal da doença hepática. Por essa razão, todos apresentam, teoricamente, risco de mortalidade devido a complicações da doença durante o período de espera pela cirurgia. A questão é agravada pela grande demanda pelo tratamento. Em 2001, foram realizados 243 Tx no Estado de São Paulo ao passo que, em maio de 2002, existiam 1.985 candidatos inscritos no cadastro da Secretaria de Saúde ${ }^{18}$. Isto significa que a oferta de órgãos é suficiente para atender apenas $12 \%$ da necessidade atual. Devido a esta desproporção, a mortalidade na lista de espera tem crescido progressivamente.

Uma outra peculiaridade do transplante de fígado é a heterogeneidade dos pacientes em lista de espera. Em alguns casos agudos e mais raros, como os portadores de hepatite fulminante, a expectativa de vida é de poucas horas ou dias. Nesses casos a priorização é prevista pela legislação brasileira e aceita consensualmente. Já na grande maioria dos transplantes, indicados por doenças crônicas, a valorização da heterogeneidade dos candidatos determina controvérsias. Alguns, considerando a disparidade existente em relação à expectativa de vida, defendem a priorização dos mais graves como forma de reduzir a mortalidade na lista de espera. Apesar de aparentemente lógica, a proposta implica vários inconvenientes e riscos. Um deles é a falta de critérios objetivos para a definição de gravidade, o que poderia permitir priorizações indevidas determinadas por falhas de julgamento ou mesmo por influência de fatores não médicos ${ }^{15}$.

Uma tentativa de contornar esta crítica é a adoção da escala MELD ${ }^{11}$. Este modelo tem algumas vantagens em relação à classificação CTP, que é classicamente utilizada para avaliação de gravidade em doenças hepáticas: (1) utiliza como variáveis apenas dados laboratoriais, o que confere ao modelo maior objetividade e reprodutibilidade. A etiologia da doença, o único parâmetro do modelo que requer interpretação, não foi considerada nesse estudo; (2) fornece valores intervalares contínuos, sem limites superior ou inferior; (3) como os coeficientes provêm de estudos estatísticos, o peso é dado a cada variável de acordo com a sua importância relativa ${ }^{11}$.

Apesar dessas vantagens, a escala MELD tem várias limitações. Uma delas é a falta de padronização dos métodos laboratoriais entre as diferentes instituições, o que determina que amostras de um mesmo paciente possam fornecer resultados distintos de $\mathrm{BT}$ e $\mathrm{Cr}$. Outra, diz respeito à situação de pacientes com doenças colestáticas, que podem ser indevidamente priorizados devido às elevadas concentrações séricas de BT que apresentam. Além de favorecer alguns doentes, a escala não contempla alguns outros, como os portadores de carcinoma hepatocelular $(\mathrm{CHC})$, polineuropatia amiloidótica familiar (PAF) ou com síndrome hepatopulmonar (SHP), nos quais a mortalidade na lista não está relacionada diretamente com o grau de insuficiência hepática. Para evitar esta distorção, a UNOS resolveu utilizar critérios arbitrários para alocação de enxertos nesses pacientes: casos de SHP ou de PAF recebem uma pontuação MELD que os permite serem transplantados dentro de três meses; casos de CHC recebem uma pontuação MELD equivalente a uma probabilidade de óbito estimada dentro de três meses. Essa probabilidade foi arbitrada em $15 \%$, para pacientes com tumores menores que $2 \mathrm{~cm}$, e em $30 \%$ para pacientes com tumores maiores ou com tumores múltiplos ${ }^{19}$. Embora estes critérios estejam em vigor, podem ser criticados por se basearem no conhecimento ainda insuficiente da história natural do $\mathrm{CHC}^{4,17}$.

Independentemente dos comentários anteriores, deve-se considerar, também, que os casos mais graves podem apresentar maior risco de mortalidade não só durante a espera mas também após o transplante. Assim, é possível que a priorização desses casos não reduza a mortalidade mas apenas a transfira para depois da cirurgia, com conseqüente desperdício de recursos e de órgãos. Ao mesmo tempo, é importante perceber que pacientes menos graves e inicialmente com melhor prognóstico pós-operatório, ao serem preteridos seriam submetidos a uma espera adicional e conseqüente agravamento da doença ${ }^{15}$.

Neste trabalho, procurou-se esclarecer o efeito 
da pontuação MELD na mortalidade após o Tx eletivo com doador cadáver. Por essa razão, foram excluídos os transplantes com doador vivo e os casos emergenciais. Os casos de PAF foram mantidos no estudo pois correspondem, no Brasil, a uma parcela significativa dos pacientes que aguardam um enxerto? $\mathrm{Na}$ presente casuística, representaram $8,9 \%$ dos casos.

Neste trabalho, as valores da escala MELD nos pacientes que foram transplantados se dispersaram pouco da média, que foi de 14,36 . Os percentis 25,50 e 75 foram, respectivamente, 9,96, 13,89 e 17,42. Assim, apesar de alguns pacientes terem apresentados valores extremos, tais como 6,08 e 50,33, mais da metade da amostra apresentou um valor de MELD inferior a 20. É possível que essa distribuição esteja subestimando o MELD médio da população que compõe a lista de espera, uma vez que pacientes com MELD mais elevado podem ter falecido antes do Tx, não tendo sido incluídos no estudo. Nos EUA, alguns dados sugerem que a maioria da população que falece na lista de espera apresenta MELD na faixa entre 25 e $30^{17}$.

Devido à pequena dispersão dos valores do MELD na casuística, a diferença entre os valores médios nos grupos A e B é de cerca de 10 pontos. Mesmo com essa distância relativamente pequena, houve diferença significante na sobrevida dos dois grupos. A análise de regressão de Cox encontrou um risco relativo de mortalidade quase duas vezes maior no grupo com MELD mais elevado. $\mathrm{Na}$ análise de regressão logística, foi encontrado um odds ratio para o MELD de 1,052, em relação à mortalidade nos primeiros 6 meses após o Tx. Isto significa que para cada aumento de uma unidade na pontuação MELD ocorre um risco 1,052 vezes maior de óbito nesse período. $O$ efeito desta interação pode ser apreciado graficamente na Figura 4. Note que a probabilidade de óbito aproximadamente duplica para cada incremento de 15 pontos na escala MELD.

Diferente de nossos resultados, Brown et al. ${ }^{3}$ estudando 42 pacientes, não encontraram associação entre os valores de MELD e a sobrevida após o Tx. Esse achado negativo pode estar relacionado à inclusão apenas de casos mais graves (UNOS 2-A), ou ao tamanho pequeno da amostra, o que pode ter determinado um erro de tipo 2.

O presente estudo estimula dois desdobramentos imediatos. O primeiro é a investigação da distribuição dos valores de MELD na população em lista de espera. Como os enxertos disponíveis permitem transplantar a cada ano cerca de $12,5 \%$ dos candidatos, o conhecimento do percentil $87,5 \%$ da lista de espera indicaria o MELD esperado na população transplantada, caso fossem priorizados os pacientes mais graves. Desta forma, seria possível realizar estimativas mais precisas do impacto causado pela alocação de enxertos através dessa escala. Na nossa casuística, este valor corresponde a 22,5. É provável que este limite seja bem maior na população que aguarda na fila de espera, determinando um impacto potencialmente expressivo na sobrevida. Outro desdobramento deriva da possibilidade dos pacientes com valores mais elevados de MELD, além da maior probabilidade de óbito, também ficarem internados por mais tempo ou terem mais complicações. Assim, seria interessante investigar o efeito da pontuação na utilização de recursos hospitalares.

Conclui-se que maiores pontuações dos receptores pela escala MELD estão associadas a maior mortalidade de pacientes e enxertos após o transplante de fígado.

Fernandes JH, Millan LS, Massarollo PCB, Mies S. The effect of Meld on the post liver transplantation mortality rate. Rev Med (São Paulo) 2002 jan./dez.;81(1/4):15-21.

ABSTRACT: The high mortality rate on the waiting list for liver transplantation (Tx) has stimulated the use of criteria of disease severity for graft allocation. The aim of this study is to asses the effect of MELD (Model for End-Stage Liver Disease) on the post Tx mortality rate. We have reviewed 237 cases of cadaveric donor elective Tx performed between $1^{\text {st }} / \mathrm{Nov} / 95$ and $30^{\text {th }} / \mathrm{Jul} / 01$. In logistic regression it was found a significant interaction between MELD score and 6-month mortality after Tx (odds ratio $=1.052 ; 95 \% \mathrm{Cl}=1.015$ a $1.090 ; \mathrm{p}=0.02$ ). In order to study the effect of the MELD score on the actuarial survival, the patients have been divided into two groups: Group $A(n=126)$, constituted by patients with MELD below the global mean score (14.4); and Group B $(n=111)$, constituted by patients with MELD score above this value. There is a statistically significant difference between the survival rate in the two groups $(p=0.0374)$. Cox proportional regression analysis indicated that group B patients are 1.65 times more likely to die (risk ratio $=1.6523 ; 95 \% \mathrm{Cl}=1.0240$ a 2.6662).

KEYWORDS: Liver transplantation/mortality; Scales; Organ procurement/standards. 


\section{REFERÊNCIAS}

1. Azoulay D, Samuel D, Castaing D, Adam R, Adam D, Said G, Bismuth H. Domino liver transplants for metabolic disorders: experience with familial amyloidotic polyneuropathy. J Am Coll Surg 1999;189:584-93.

2. Bittencourt PL, Couto, CA, Farias $A Q$, Marchiori $P$, Massarollo PCB, Mies, S. Results of liver transplantation for familial amyloid polyneuropathy type I in Brazil. Liver Transplant 2002;8:34-9.

3. Brown RS Jr, Kumar KS, Russo MW, Kinkhabwala M, Rudow DL, Harren P, Lobritto S, Emond, JC. Model for end-stage liver disease and child-turcotte-pugh score as predictors of pretransplantation disease severity, posttransplantation outcome, and resource utilization in United Network for Organ Sharing status 2A patients. Liver Transplant 2002;8:278-84.

4. Cheng SJ, Freeman RB, Wong JB. Predicting the probability of progression-free survival in patients with small hepatocellular carcinoma. Liver Transplant 2002;8:323-8.

5. Christensen R. Log-linear models and logistic regression. 2nd. ed. New York: Springer-Verlag; 1997.

6. Cox, D.R. Regression models and lifetables. J R Stat Soc (Series B) 1972;34:187-202.

7. Dienstag LD. Liver transplantation. In: Braunwald E, Fauci AS, Kasper DL, Hauser SL, Longo DL, Jameson JL. Harrison's principles of internal medicine. 15th. ed. New York: McGraw-Hill; 2001. v.2, p.1770-6.

8. Entidades defendem manutenção de "fila única" para fígado. J CREMESP 2001;18:6.

9. Freeman RB, Rohrer RJ, Katz E, Lewis, WD, Jenkins $R$, Cosimi $A B$, et al. Preliminary results of a liver allocation plan using a continuous medical severity score that de-emphasizes waiting time. Liver
Transplant 2001;7:173-8.

10. Friedman LM, Furberg CD, DeMets DL. Survival analysis. In: Friedman LM, Furberg CD, DeMets DL. Fundamentals of clinical trials. 3rd. ed. New York: Spriger-Verlag; 1998. p.233-45.

11. Kamath PS, Wiesner RH, Malinchoc M, Kremers W, Therneau TM, Kosberg CL, et al. A model to predict survival in patients with end-stage liver disease. Hepatology 2001;33:464-70.

12. Kaplan EL, Meier P. Nonparametric estimation from incomplete observations. J Am Stat Assoc 1958;53:457-81.

13. Nair S, Verma S, Thuluvath PJ. Pretransplant renal function predicts survival in patients undergoing orthotopic liver transplantation. Hepatology 2002;35:1179-85.

14. Raia S, Massarollo PCB, Baía CES, Fernandes AONG, Lallée MP, Bittencourt $P$, et al. Transplante de fígado "repique": receptores que também são doadores. J Bras Transplant 1998;1:222.

15. Raia S, Massarollo PCB. Doação de órgãos. In: Gayotto LCC, Alves VAF. Doenças do fígado e das vias biliares. São Paulo: Atheneu; 2001. v.2, p.111320.

16. Raia S, Nery JR, Mies S. Liver transplantation from live donors. Lancet 1989;2(8661):497.

17. Roberts JP. Priorization of patients with liver cancer within the MELD system. Liver Transplant 2002;8:329-30.

18. Secretaria de Estado da Saúde de São Paulo. Disponível em: URL: http://www.saude.sp.gov.br. Acesso em: 4 jul 2002.

19. United Network for Organ Sharing. [Cited 2002 Jun 1]. Available from: URL: http://www.unos.org. 